EPJ Web of Conferences 81, 05015 (2014)

DOI: 10.1051/epjconf/20148105015

(C) Owned by the authors, published by EDP Sciences, 2014

\title{
Analysis of antikaon-induced cascade production
}

\author{
Benjamin C. Jackson ${ }^{1, a}$, Helmut Haberzett $\left.\right|^{2}$, Yongseok Oh ${ }^{3,4}$, and Kanzo Nakayama ${ }^{1,5}$ \\ ${ }^{1}$ Department of Physics and Astronomy, The University of Georgia, Athens, GA 30602, USA \\ ${ }^{2}$ Institute for Nuclear Studies and Department of Physics, The George Washington University, Washington, \\ DC 20052, USA \\ ${ }^{3}$ Department of Physics, Kyungpook National University, Daegu 702-701, Korea \\ ${ }^{4}$ Asia Pacific Center for Theoretical Physics, Pohang, Gyeongbuk 790-784, Korea \\ ${ }^{5}$ Institut für Kernphysik and Center for Hadron Physics, Forschungszentrum Jülich, 52425 Jülich, Germany
}

\begin{abstract}
In preparation for forthcoming experiments on multi-strangeness baryon production at JPARC, we analyze the general features of $\Xi$ production in antikaon-induced reactions. A simple model is applied to this reaction; problems with retaining $s-u$ symmetry are addressed with a generalized contact term. Existing data are reproduced and any hyperon resonance features are extracted.
\end{abstract}

\section{Introduction}

Hadron spectroscopy is an essential part of the investigation to understand the non-perturbative regime of Quantum Chromodynamics (QCD). To compare various theoretical results with the experimental data, a reliable reaction theory capable of identifying resonances and extracting their parameters is required.

Recently, the study of multi-strangeness baryons has started to attract renewed interest. On the experimental side, cascade processes are to be studied at JLab and the FAIR facility at GSI. At JPARC, in particular, $\Xi$ production will be studied by the basic two-body process $\bar{K} N \rightarrow K \Xi$; other plans include studying the $\pi N \rightarrow K K \Xi$ reaction as well as $\Omega$ production [1,2].

In the present work, we concentrate on the two-body reaction

$$
\bar{K}(q)+N(p) \rightarrow K\left(q^{\prime}\right)+\Xi\left(p^{\prime}\right),
$$

where, in particular, $\Xi$ is the cascade ground state with $S=-2$. The existing data are rather limited and suffer from large uncertainties and early theoretical attempts to understand the above reaction are very few. Some of the model-independent aspects of reaction (1) have been studied recently by the present authors $[3,4]$. Here, we present a model-dependent analysis of the existing data based on the effective Lagrangian approach that includes a phenomenological contact amplitude which accounts for the rescattering contributions and/or unknown (short-range) dynamics that have not been included explicitly into the model.

For lack of space, we defer a more comprehensive listing of pertinent experimental and theoretical references to a later publication [5].

\footnotetext{
ae-mail: ben@physast.uga.edu
} 


\section{Model Description}

The reaction amplitude $T$ describing the two-body process like the reaction (1) is, in general, given by the Bethe-Salpeter equation

$$
T=V+V G_{0} T
$$

where $V$ stands for the (two-body) meson-baryon irreducible (Hermitian) driving amplitude and $G_{0}$ the meson-baryon propagator. It can be recast into the pole part, $T^{\mathrm{P}}$, and the non-pole part, $T^{\mathrm{NP}}$, as

$$
T=T^{\mathrm{P}}+T^{\mathrm{NP}}, \quad \text { with } \quad T^{\mathrm{NP}}=V^{\mathrm{NP}}+V^{\mathrm{NP}} G_{0} T^{\mathrm{NP}}
$$

where $V^{\mathrm{NP}} \equiv V-V^{\mathrm{P}}$ denotes the one-baryon irreducible (non-pole) part of the driving amplitude $V$.

In the present work, we shall make the following approximations to the reaction amplitude in Eq. (3). First, we approximate the pole part of the reaction amplitude, $T^{\mathrm{P}}$, by the $s$-channel Feynman amplitude $M_{s}$ specified by effective Lagrangians and phenomenological Feynman propagators; their dressed coupling constants, dressed baryon masses, and the corresponding widths are the parameters to be adjusted to reproduce the experimental data. The details of the Lagrangians and propagators used in this calculation can be found in Refs. [6, 7].

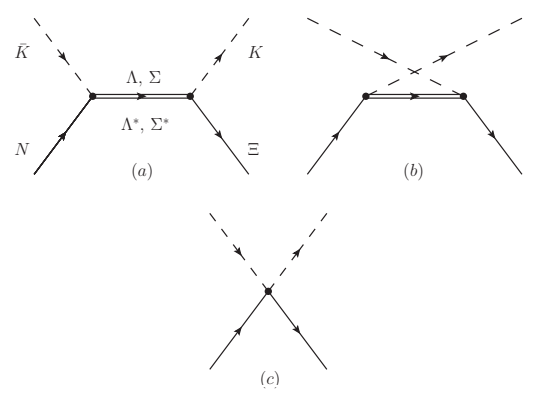

Figure 1. Diagrams for (a) $s$-channel and (b) $u$-channel exchanges of hyperons and hyperon resonances; (c) contact diagram (see Eq. 5).
Second, the non-pole part of the reaction amplitude, $T^{\mathrm{NP}}$, is approximated as follows.

(i) Since there is no meson-exchange $t$-channel process in the present reaction, unless the exchanged meson is an exotic one with strangeness quantum number $S=2, V^{\mathrm{NP}}$ of the reaction is approximated by the $u$-channel Feynman amplitude, $M_{u}$.

(ii) The rescattering term $V^{\mathrm{NP}} G_{0} T^{\mathrm{NP}}$ in $T^{\mathrm{NP}}$ of Eq. (3), and other effects not included explicitly in the present approach, are accounted for by a phenomenological contact term, $M_{c}$, specified below, in Eq. (5).

With these approximations, the reaction amplitude in the present work is given by

$$
T=M_{s}+M_{u}+M_{c},
$$

where $M_{s}$ and $M_{u}$ are the $s$ - and $u$-channel Feynman diagrams with the ground state hyperons ( $\Lambda$ and $\Sigma$ ) as well as some of the $S=-1$ hyperon resonances in the intermediate state. Figure 1 shows a diagrammatic representation of $M_{s}, M_{u}$, and $M_{c}$.

The contact amplitude $M_{c}$ is decomposed in terms of the spin-flip and spin-non-flip amplitudes, each expanded in partial-waves. ${ }^{1}$ Following the essential idea of Ref. [8] for the phenomenological contact term $M_{c}$, the spin amplitudes in the above equation are parametrized as

$$
M_{c \frac{1}{2} \frac{1}{2}}=M_{c-\frac{1}{2}-\frac{1}{2}}=\sum_{L^{\prime}, T} a_{L^{\prime}}^{T}\left(\frac{p^{\prime}}{\Lambda_{S}}\right)^{L^{\prime}} \exp \left(-\alpha_{L^{\prime}}^{T} \frac{p^{\prime 2}}{\Lambda_{S}^{2}}\right) P_{L^{\prime}}(\theta) \hat{P}_{T},
$$

\footnotetext{
${ }^{1}$ There are in total four spin matrix elements to describe the reaction (1). However, only two of them, corresponding to the spin-non-flip and spin-flip processes, are independent due to the reflection symmetry about the reaction plane for parity conserving processes. See Ref. [4] for more detailed discussions.
} 


$$
M_{c \frac{1}{2}-\frac{1}{2}}=-M_{c-\frac{1}{2} \frac{1}{2}}=\sum_{L^{\prime}, T} b_{L^{\prime}}^{T}\left(\frac{p^{\prime}}{\Lambda_{S}}\right)^{L^{\prime}} \exp \left(-\alpha_{L^{\prime}}^{T} \frac{p^{\prime 2}}{\Lambda_{S}^{2}}\right) P_{L^{\prime}}^{1}(\theta) \hat{P}_{T},
$$

with $a_{L^{\prime}}^{T}, b_{L^{\prime}}^{T}$, and $\alpha_{L^{\prime}}^{T}$ being constants to be fitted and $p^{\prime}$ is magnitude of the center-of-mass threemomentum of the final-state kaon and $\Lambda_{S}=1 \mathrm{GeV}$. The summation extends over all angular momenta $L^{\prime}$ and isospin channels $T$, with $\hat{P}_{T}$ being the projector for the latter. The exponential factor in Eq. (5) is simply a damping factor to suppress the high-momentum behavior introduced by $p^{\prime L^{\prime}}$. For details, we refer to Ref. [8].

It should be noted that our phenomenological contact term $M_{c}$ can only account for effects that give rise to a smooth energy dependence. Effects such as due to dynamically generated resonances and/or channel couplings [9-12] that give rise to a strong variation of the amplitude as a function of energy cannot be taken into account by the contact term.

\section{Results}

In our attempts to reproduce the data displayed in Figs. 2 and 3, all 3- and 4-star hyperon resonances were considered. Also, a test was performed to determine the minimum number of partial waves needed in our contact amplitude. Here we present results of our model which included $\Lambda(1116)$, $\Lambda(1890), \Sigma(1193), \Sigma(1385), \Sigma(2030)$ and partial waves up to $L^{\prime} \leq 2$ in the contact term.

As can be seen in Fig. 3, the $K^{-}+p \rightarrow K^{+}+\Xi^{-}$reaction shows a backward peaking cross section, while $K^{-}+p \rightarrow K^{0}+\Xi^{0}$ shows less of this behavior for all but the highest energy. This backward peak may indicate a strong $u$-channel exchange. It should be noted that $u$-channel $\Lambda$ exchange is forbidden in the $K^{-}+p \rightarrow K^{0}+\Xi^{0}$ reaction due to charge conservation and that the $u$-channel diagrams give largest contributions for low-mass resonances. This may be an indication that sub-threshold $\Lambda u$ channel contributions are dominating the $\Xi^{-}$production amplitude. In our attempts to model this behavior, without a contact term, serious problems were encountered, mainly the result of a growing total cross section as a function of $W$. This was the case for all resonances with $J \geq \frac{3}{2}$. Recent efforts
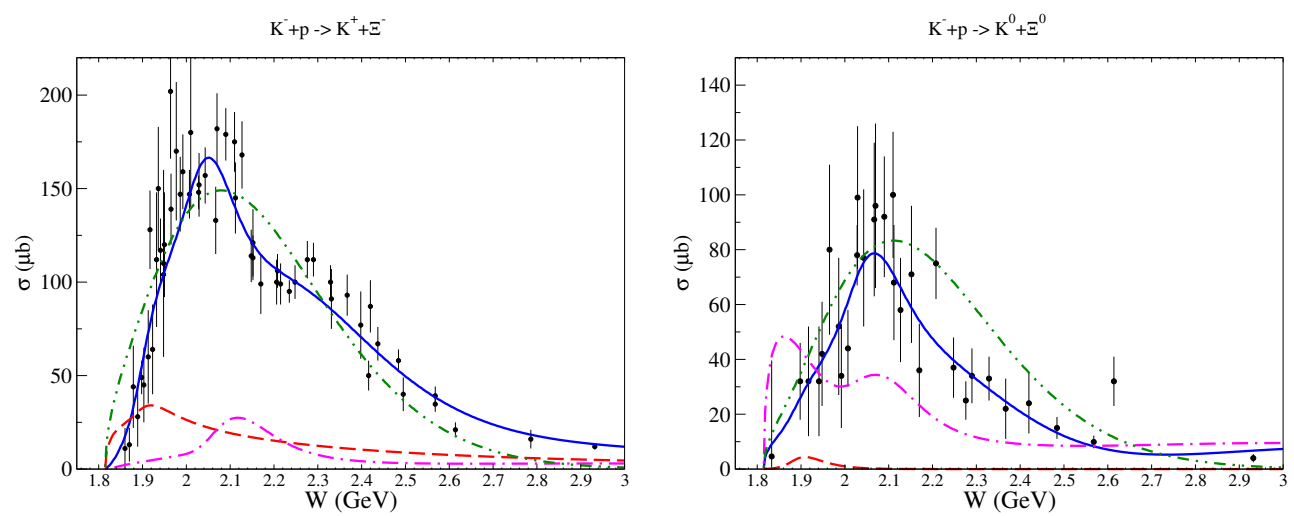

Figure 2. Total cross section for the $K^{-}+p \rightarrow K^{+}+\Xi^{-}$and $K^{-}+p \rightarrow K^{0}+\Xi^{0}$ reactions. The solid blue line represents the result of the full calculations of the present model. The red dashed line shows the combined contribution of $\Lambda$ hyperons. The magenta dash-dotted line shows the combined contribution of $\Sigma$ hyperons. The green dash-double-dotted line gives the contribution from the contact term. The experimental data are adopted from Ref. [13]. 

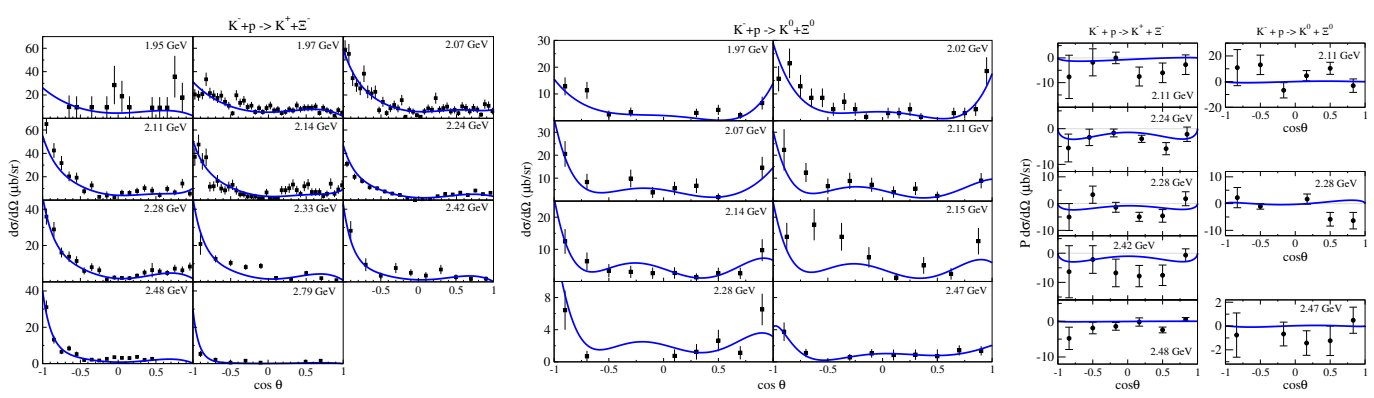

Figure 3. The left and center figures show kaon angular distributions in the center-of-mass frame for $K^{-}+p \rightarrow$ $K^{+}+\Xi^{-}$(left figure) and for $K^{-}+p \rightarrow K^{0}+\Xi^{0}$ (center figure). The right figure shows $\frac{d \sigma}{d \Omega} P$ for both the $K^{-}+p \rightarrow K^{+}+\Xi^{-}$and $K^{-}+p \rightarrow K^{0}+\Xi^{0}$ reactions. The blue lines represent the full model results. The numbers in the upper right corners correspond to the centroid total energy of the system, $W$, in the energy bins. The experimental data are adopted from Ref. [13].

of other groups $[13,14]$ to remedy this situation differ but both rely on form-factors to quell the growing $u$-channel contribution. In both attempts, $s$ - $u$ crossing symmetry is violated. Our approach uses the contact term, $M_{c}$, to model the effect of these low-mass resonances in a simple, efficient and effective manner while maintaining $s-u$ crossing symmetry.

\section{Acknowledgements}

We are grateful to D. A. Sharov for providing us with the digitized version of the data used in the present work. This work is supported by the National Research Foundation of Korea funded by the Korean Government (Grant No. NRF-2011-220-C00011). The work of K.N. was also supported in part by the FFE- COSY Grant No. 41788390 .

\section{References}

[1] J. K. Ahn, J. Korean Phys. Soc. 49, 2276 (2006).

[2] H. Takahashi, Nucl. Phys. A 914, 553 (2013).

[3] K. Nakayama, Y. Oh, and H. Haberzettl, Phys. Rev. C 85, 042201(R) (2012).

[4] B. Jackson, Y. Oh, H. Haberzettl, and K. Nakayama, Phys. Rev. C 89, 025206 (2014).

[5] B. Jackson, Y. Oh, H. Haberzettl, and K. Nakayama, in preparation.

[6] K. Nakayama, Y. Oh, and H. Haberzettl, Phys. Rev. C 74, 035205 (2006).

[7] J. K. S. Man, Y. Oh, and K. Nakayama, Phys. Rev. C 83, 055201 (2011).

[8] K. Nakayama and W. G. Love, Phys. Rev. C 72, 034603 (2005).

[9] M. Döring and K. Nakayama, Phys. Lett. B 683, 145 (2010).

[10] A. Ramos and E. Oset, Phys. Lett. B 727, 287 (2013).

[11] H.-Y. Ryu, A. I. Titov, A. Hosaka, and H.-C. Kim, Prog. Theor. Exp. Phys. 2014, 023D03 (2014).

[12] H. Ryu, A. Hosaka, H.-C. Kim, and A. I. Titov, Int. J. Mod. Phys. Conf. Ser. 26, 1460055 (2014).

[13] D. A. Sharov, V. L. Korotkikh, and D. E. Lanskoy, Eur. Phys. J. A 47, 109 (2011).

[14] R. Shyam, O. Scholten, and A. W. Thomas, Phys. Rev. C 84, 042201 (2011). 